ITC 2/49

Information Technology and Control

Vol. 49 / No. 2/2020

pp. 260-274

DOI 10.5755/j01.itc.49.2.24111
On Improving Reliability in Multicast Routing

Protocol for Wireless Sensor Network

Received 2019/08/28

Accepted after revision 2020/01/14

HOW TO CITE: Sampradeepraj, T., Raja, S. P. (2020). On Improving Reliability in Multicast Routing Protocol for Wireless Sensor Network. Information Technology and Control, 49(1), 260-274. https://doi.org/10.5755/j01.itc.49.2.24111

\title{
On Improving Reliability in Multicast Routing Protocol for Wireless Sensor Network
}

\section{T. Sampradeepraj}

Department of Computer Science and Engineering, Sethu Institute of Technology, Madurai, Tamilnadu, India; phone: +919095054161; e-mail: sampradeepraj@gmail.com

\section{S. P. Raja}

Department of Computer Science and Engineering, Vel Tech Rangarajan Dr. Sagunthala R\&D Institute of Science and Technology, Avadi, Chennai, Tamilnadu, India; phone:+919486181212; e-mail: avemariaraja@gmail.com

Corresponding author: sampradeepraj@gmail.com

Multicast is a process, which sends data to many objects. Multicast routing is an important challenging task in group communication in Wireless Sensor Network (WSN). Reliable multicasting is necessary for efficient group communication in WSN. In this paper, an efficient algorithm Minimum Connected Dominating Set (MCDS) is used to form a virtual backbone (Forwarding Group) of the network. The MCDS aims to minimizing the number of nodes, where few nodes are participating in forwarding the multicast data. Random Linear Network Coding (RLNC) has a great potential to improve the throughput and reliability by reducing the multiple transmissions in wireless network. The objective of this paper is to propose an efficient reliable multicast routing protocol named as RLNMCDS-ODMRP (Random Linear Network Coding over Minimum Connected Dominating Set in On-Demand Multicast Routing Protocol) by using two state-of-arts techniques MCDS and RLNC in ODMRP for WSN. Experimental results and performance analysis show that the proposed protocol delivers multicast data in high reliable and outperforms the classical ODMRP that use MCDS or RLNC.

KEYWORDS: Wireless Sensor Networks, ODMRP, MCDS, RLNC. 


\section{Introduction}

Wireless Sensor Network is a type of wireless network, which consist number of sensor nodes to measure a physical quantity and monitor the environmental or physical conditions [3]. WSN has many applications such as military application, traffic surveillance, environment monitoring, building structures monitoring, habitat monitoring, information gathering, pollution monitoring and wildfire detection [3]. Multicasting is an efficient method of sending a data to many receivers at a same time, which has many applications such as multimedia conference meetings and in battle field for multicast tactical messages [8].

In this paper, reactive and on demand [37] multicast routing protocol called ODMRP [27] is chosen for multicasting data in WSN. Reliability is an important issue to prove end-to-end successful delivery of data in WSN, since there is a possibility to failure of data and unstable of network. It is important to develop high reliable multicast routing protocol. Also, network backbone formation and channel capacity are some issues [45] to improve the reliability, To solve these issues two most popular techniques were used, they are, MCDS and RLNC.

In Connected Dominating Set (CDS), dominating nodes are connected to form a virtual backbone (Forwarding Group) and reduce the redundant transmission in the network [44]. Dominating Set (DS) nodes are realying the messages, maintain routing tables,reduce the communication cost, reduce the redundant traffic, localize the routing information, save storage space and it provides reliable connectivity between the nodes. The MCDS is also a connected dominating set with minimum number of elements [9], which is NP-Hard to find a minimum sized connected dominating set [13]. In real time environment, the virtual backbone of the network as small as possible, in order to decrease the protocol overhead, to save life time, energy consumption and cost of construction etc. Hence, it is required to form a minimum sized CDS. In WSN, constructing minimum sized CDS is an important issue because it reduces unnecessary multicast message transmission. Finding MCDS of the network is a promising approach. MCDS is constructed based on computation of Convex Hull $(\mathrm{CH})$ in Unit Disk Graph (UD Graph) [36]. Limited number of sensor nodes are participated for multicast trans- mission in MCDS, which improves the reliability in the network.

Network Coding (NC) is a technique which improves throughput and reliability by reducing the number of transmissions and save the bandwidth in wireless network [2]. RLNC is also a type of network coding where forwarding nodes add some packets with original data using mathematical operations [35].

\subsection{Motivation and Justification}

Recently, some researchers have constructed virtual backbone by using MCDS alone for multicast operation and to improve performance of network [4, $7,38]$. In general, MCDS can be constructed in two ways either in global or local information of network and centralized or distributed way respectively. Due to the characteristics of WSN, it is hard to obtain and maintain global information of network, Moreover, MCDS construction is inefficient for a single node [30]. Therefore, MCDS is constructed based on local information and distributed way in the proposed protocol RLNMCDS-ODMRP.

Javad, A.T. et al. [45] proposed Weighted Steiner Connected Dominating Set (WSCDS) for multicast routing in MANET. Shuai Wang et al. [48] explored broadcast protocols in energy minimal for wireless ad hoc networks by using network coding over connected dominating set (CDS). Zhao Zhang et al. [52] introduced polynomial time approximation scheme (PTAS) to improve the running time and for minimum CDS in WSN. Hongwei Du et al. [9] reduced routing cost and improved the load balance by PTAS for MCDS in WSN. Xiaoyan Kui et al. [26] investigated energy balanced CDS problem in constructing network backbone in WSN. Dennis Lisiecki et al. [33] enhanced the energy efficiency by exploiting connected dominating to reduce the nubmer of transmissions in WSN. Xuemei Sun et al. [42] proposed an algorithm to solve maximum independent set (MIS) to expand the dominating point in MCDS. Xin Bai et al. [6] considered the upper bounds of MIS to design an MCDS algorithm for 3D heterogeneous ad hoc networks. Md. Sadiqur Rahman Sohel et al. [41] developed contention aware connected dominant set (CACDS) algorithm also provided mathematical analysis of contention for connected Internet of Things (IoT) devices. 
Using routing, the multicast data cannot be communicated to destination nodes at a time. However, if the member nodes are performing linear network coding operations in addition to routing, the multicast data can be communicated to destination nodes at a time and achieves the maximum capacity of multicast network [11]. Ahlswede [2] illustrated this through famous "butterfly network". Therefore, RLNC is essential to communicate a sender to multiple receivers at same time. Most of the researchers has been applied RLNC alone for various multicast applications and increases the capacity of the network in MANET and Wireless Mesh Network (WMN) [43, 51, 24, 31, 46, 32]. In which, RLNC has great potential for improving performance in throughput, reliability and minimize the transmission delay in MANET.

Kerim Fouli et al. [16] described both block and sliding window RLNC for reliable data transport. Evgeny Tsimbalo et al. [47] addressed the performance analysis of successful decoding at nodes under RLNC. Arash Ghafouri et al. [12] improving the ODMRP protocol performance using power-based method. Mallapur, S.V. et al. [34] proposed to build a reliable path between nodes to recover lost packets during communication. WSN differ from the MANET in terms of network topology, performance metrics, clustering, traffic patterns, hardware, mobility, communication technology and their amount of available memory. These differences are considered in RLNC and different approaches have been proposed by researchers for WSN [23, 14, 39, 48, 29]. RLNC provides loss recovery in low quality wireless links and economical path diversity in WSN [20].

S. Katti etal. [22] proposed a new architecture (COPE) to forward and mix the packets by XOR operation to increase throughput. Tracey Ho et al. [17] presented a distributed RLNC for multicast in wireless networks.
Rout, $R R$ et al. [39] attempted to improve the network lifetime in WSN by considering duty cycle and network coding. Zhu et al. [53] proposed distributed and novel method to construct a 2 -reduandant multicast graph by applying network coding. Dumitrescu et al. [10] proposed a layered multicast schemes using network coding. Jaggi et al. [19] presented a deterministic polynomial time algorithm to design linear codes for directed acyclic graphs.

The proposed protocol RLNMCDS-ODMRP aims to develop efficient and high reliable multicast routing protocol. WSN protocols must be simple for both computation and communication overhead. Also, it should be implemented easily, scalable, efficient, adaptive and should be reduced redundant transmissions in various situations. The efficient protocol shoud achieve maximum throughput, reliability, packet delivery ratio, minimum end-to-end delay, security and energy efficiency, therefore all the aforementioned conditions are considered in the proposed protocol. So far, there is no work on ODMRP with RLNC over MCDS for WSN. Thus, the proposed protocol is essential to develop efficient multicast routing protocol.

\subsection{Outline of the Paper}

This paper first presents comprehensive investigation of MCDS and RLNC, also discusses their operations in detail. Second, describes implementation of the proposed protocol, which has two phases as shown in Figure 1. In the first phase, source node discovers the route and constructs the MCDS using convex hull, the source node transmitting the data by applying RLNC through the constructed MCDS in ODMRP to its receivers in the second phase. At the end of this research work, performance of proposed protocol is evaluated.

\section{Figure 1}

Outline of the Paper

Phase I




\subsection{Organization of the Paper}

The residual paper is organized as follows: Proposed methodology is given in Section 2. Section 3 converses about the experimental results. Finally, conclusion about the proposed approach is given in Section 4.

\section{Proposed Methodology}

In this paper, two most popular techniques were used, they are, (1) Minimum Connected Dominating Set, (2) Random Linear Network Coding.

\subsection{Construction of Minimum Connected Dominating Set}

The concept of the MCDS derived from graph theory [50]. The set of nodes are connected for a given graph (network) is called as CDS $[45,9,13,4,7,26,33,41]$ is shown in Figure 2, which shows that the nodes in blue connected through blue bold lines and form a MCDS, which is backbone of the network. Remaining nodes are marked in white and green node (receiver) can be reached by the blue nodes in the MCDS to reduce the redundant transmissions of multicast data [40]. MCDS is constructed from CDS using convex hull in the following steps as shown in the Figure 3.

STEP 1: Find the minimum degree vertex in CDS. Degree of vertex $\mathrm{C}$ is 3, Degree of vertex $\mathrm{E}$ is 4, Degree of vertex $G$ is 3 , Degree of vertex $F$ is 5 and Degree of vertex $\mathrm{D}$ is 4 . Now consider the minimum degree vertex C.

STEP 2: Calculate convex hull of N[C] ([ ] - closed neighborhood, ( ) - open neighborhood) as shown in Figure 4. $\mathrm{CH}(\mathrm{N}[\mathrm{C}])$ is $\{\mathrm{E}, \mathrm{C}, \mathrm{D}\}$

STEP 3: Calculate convex hull of N[i] as shown in Figure 5.

STEP 4: Check if convex hull of $\mathrm{N}[\mathrm{C}]$ is contained in $U C H(N[i])$ where $i \in \mathrm{N}(\mathrm{C})=\{\mathrm{E}, \mathrm{B}, \mathrm{D}\}$ as shown in Figure 6.

STEP 5: If Step 4 is true, then remove the vertex C from CDS and go to step1

STEP 6: Select the next minimum degree vertex i.e. G and repeat the process from step1 to step5. By above process, remove the vertex $\mathrm{G}$ and go to step 1

STEP 7: Select the next minimum degree vertex i.e. $D$
STEP 8: Calculate $\mathrm{CH}(\mathrm{N}[\mathrm{D} 1)$ and Convex hull of $\mathrm{N}$ [D] is $\mathrm{CFJ} \mathrm{CH}(\mathrm{N}[\mathrm{D}]){ }^{\circ} \phi{ }_{i}^{Y} \mathrm{CH}(\mathrm{N}[i])$

STEP 9: Calculate $\mathrm{CH}(\mathrm{N}[\mathrm{i}])$ for all $\mathrm{i} \in \mathrm{N}(\mathrm{D})=\{\mathrm{B}, \mathrm{F}, \mathrm{C}, \mathrm{J}\}$

STEP 10: Convex Hull (N[D]) is not belongs to $\bigcup C H(N[i])$ where $\mathrm{i} \in \mathrm{N}(\mathrm{D})=\{\mathrm{B}, \mathrm{F}, \mathrm{C}, \mathrm{J}\}$

STEP 11: If step 10 is false, don't remove the vertex D from CDS and go to step 1

STEP 12: Select the next minimum degree vertices $E$ and $F$, and go to step 1. Likewise two vertices $E$ and $F$ are not deleted from CDS. Finally CDS left with the vertices E, D and F. Thus, as shown in Figure 7 constructed MCDS is $\{E, D, F\}$ from $C D S$ of $\{C, E, G, F, D\}$.

\section{Figure 2}

The CDS Network

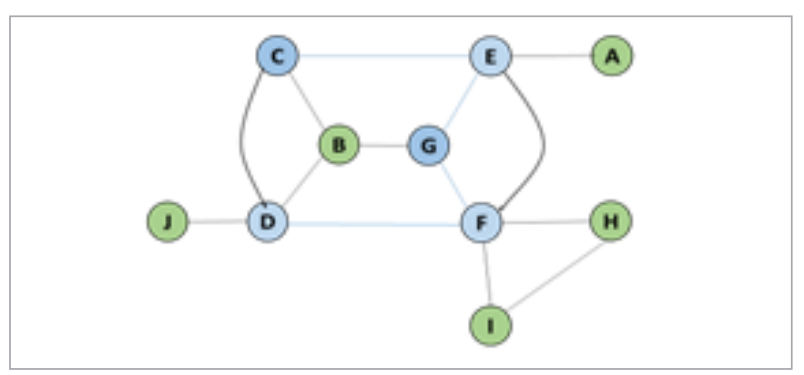

Figure 3

The MCDS Network

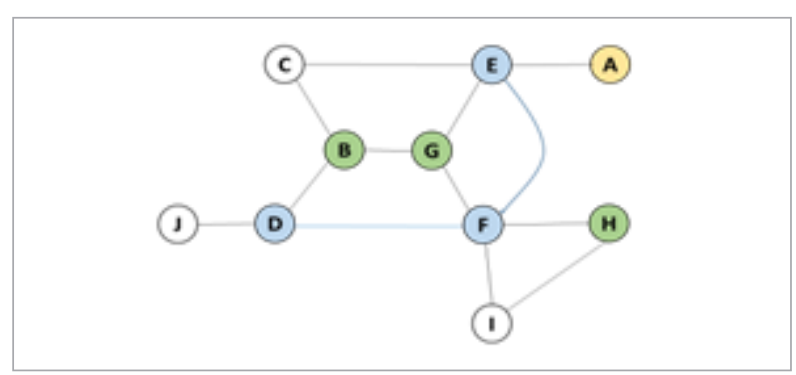

Figure 4

$\mathrm{CH}(\mathrm{N}[\mathrm{C}])$ is $\{\mathrm{E}, \mathrm{C}, \mathrm{D}\}$

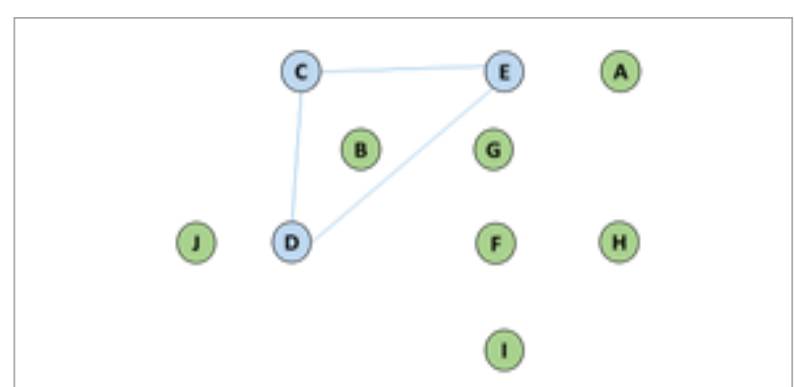


Figure 5

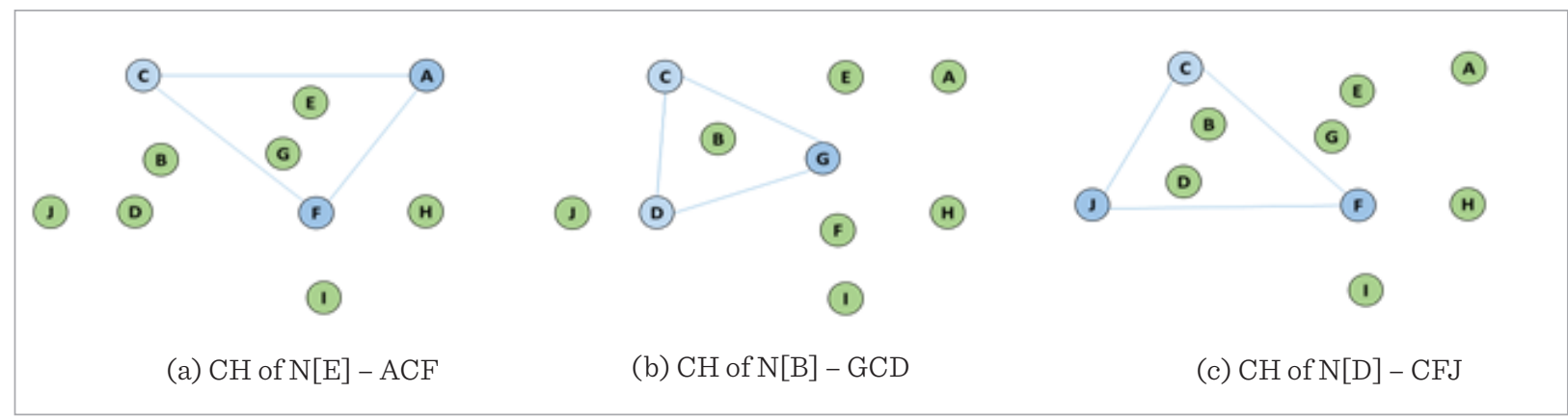

Figure 6
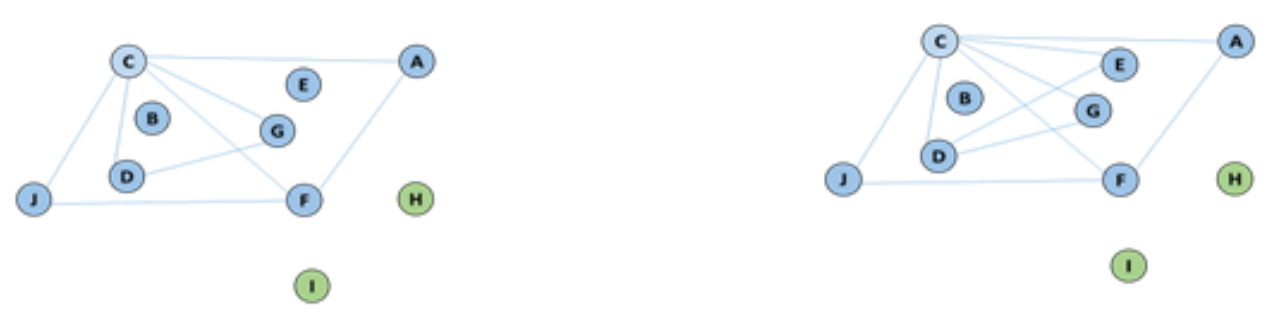
(a) $\underset{i}{\bigcup} C H(N[i])$
(b) $\mathrm{CH}(\mathrm{N}[\mathrm{C}]) \subseteq \underset{i}{\bigcup} \mathrm{C} \quad(N[i])$

Figure 7

Constructed MCDS Network

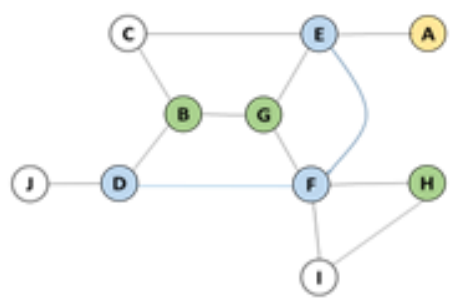

\subsection{Multicast Routing Protocol}

In this work, the selected simple and scalable reactive or on-demand routing protocol is ODMRP. Because, most of the researchers show that reactive method is better than the proactive method in many aspects such as nodes movement, network life time, self-organizing network model [27].

\subsubsection{On-demand Multicast Routing Protocol}

ODMRP is a state-of-art, mesh based and a source ini- tiated protocol, where forwarding group concept and soft state approach is used to establish and maintain a mesh structure, respectively, in a given network [27].

\subsubsection{Minimum Connected Dominating Set based Multicast}

Recently, MCDS is used to construct virtual backbone for multicasting in many wireless networks. MCDS based multicast routing is not only applied for proactive routing, it can also be applied to reactive routing, where routes are computed in on-demand. In this paper, reactive multicast routing protocol, ODMRP is considered. Figure 8 shows MCDS network with ODMRP. In the MCDS network, each dominating node keeps following information: Dominating node's membership list, routing table and forwarding node table. Adjacent node of dominating node is called as membership list. Routing table includes next-hop information of a shortest path and the distance to the specified MCDS destination. This work extends the ODMRP algorithm by adding further routing information as shown in Figure 8 (b) to be sent between MCDS nodes to a quite selective multicast process. 
Figure 8

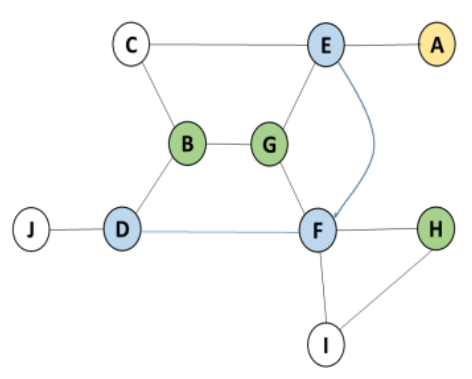

(a) Multicast data transmission through MCDS

Sender $=1$

Receiver= $3,11,12,16,20$

Forwarding Group $=4,7,8,9$

Gateway Domain member list of node 4:2, 5

Gateway routing table of node 4:

\begin{tabular}{c|cc|c}
\hline Destination & Member list & Next Hop & Distance \\
\hline 7 & $6,10,11,12$ & 7 & 1 \\
8 & $13,14,15,16$ & 8 & 1 \\
9 & $17,18,19,20$ & 7 & 2
\end{tabular}

(b) Multicast Routing information

\subsection{Random Linear Network Coding}

Recently, RLNC is emerged promising technique for various applications in wireless networks, which is used to increase the throughput of the network and reduce the multicast traffic in wireless network.

\subsubsection{Random Linear Network coding for Unicast}

In RLNC, Output data of each node is in the form of linear combination of input message. The coefficients selected for this linear combination are completely random in nature. The forwarding node adds its own packets with original packets and sends outgoing coded packets. Typically, RLNC performs three different operations [21], they are 1. Encoding, 2. Re-encoding, 3. Decoding.

From the Figure 9, the encoding process can be done at source node of the network. Re-encoding process can be done at forwarding node, which is almost similar to encoding process but the coefficients are completely newly generated. Finally, decoding process can be done at destination nodes. The encoding, re-encoding and decoding operations are implemented via matrix
Figure 9

RLNC Process

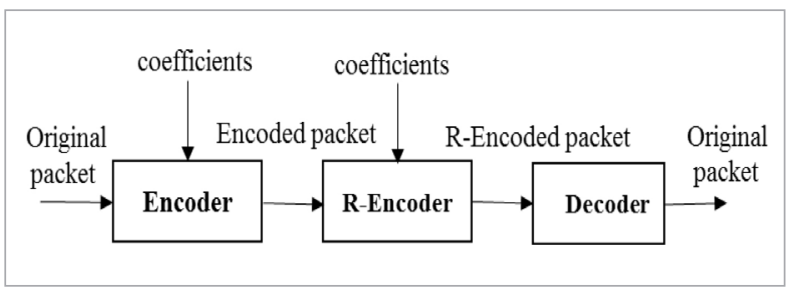

operations. First, consider the unicast network, when there is a single-source single-destination capacity (max-flow) is achievable by min-cut max-flow [33]. The basic idea of RLNC for unicast communication can be illustrated in the Figures 10-13.

\section{Figure 10}

A Simple example of RLNC for unicast in WSN

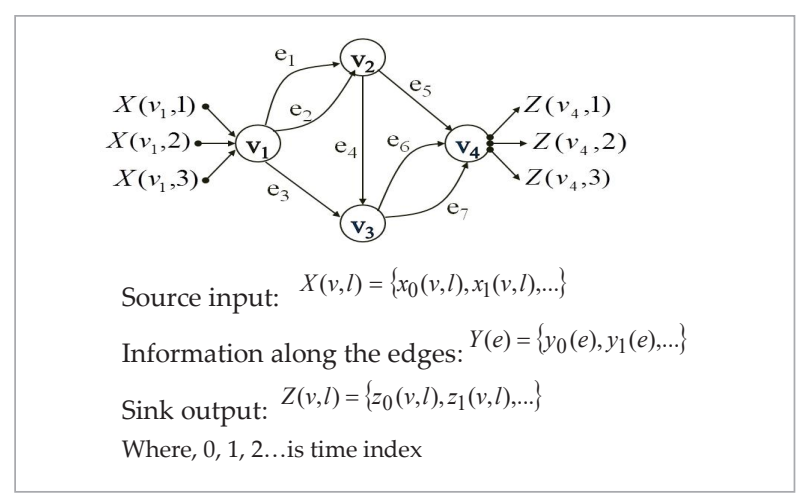

Figure 11

Encoder at Source node

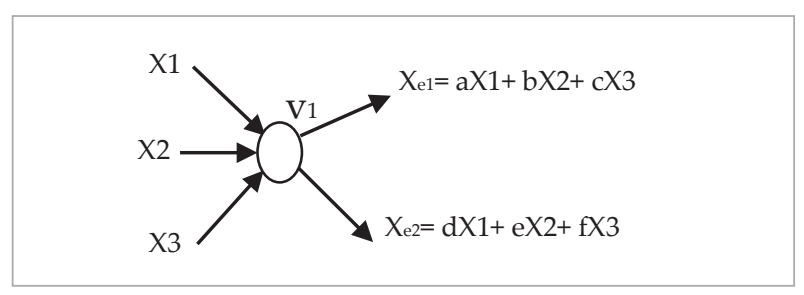

\section{Figure 12}

Re-encoder at intermediate node

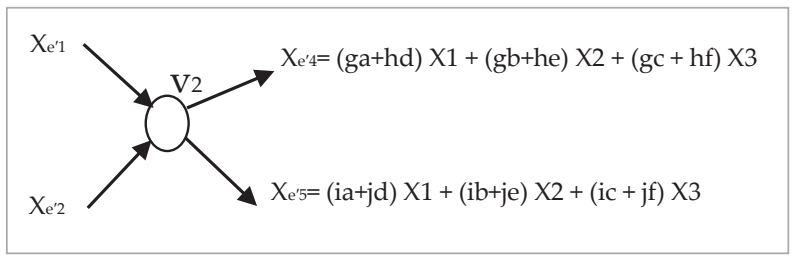


Figure 13

Decoder

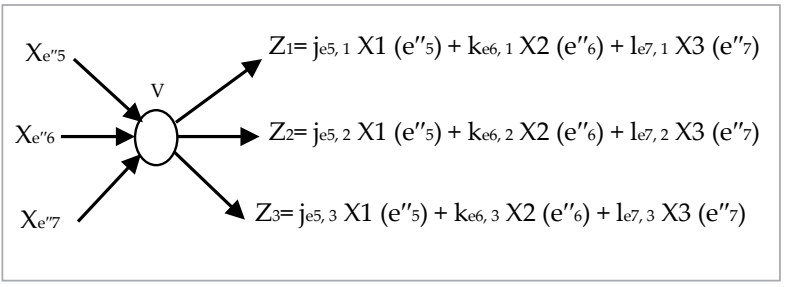

The data X1, X2 and X3 are given to node V1 as input then node (V2) received two coded packets: aX1+ $\mathrm{bX} 2+\mathrm{cX} 3$ and $\mathrm{dX} 1+\mathrm{eX} 2+\mathrm{fX} 3$ as output of node V1. In order to perform the re-encoding operation on the two received coded packets, the node (V2) generates two random coefficients $(\mathrm{g}, \mathrm{h})$ for the two coded packets to be re-encoded. The coding vector of the new re-encoded packet can be calculated as following: $\mathrm{g}(\mathrm{aX} 1+\mathrm{bX} 2+\mathrm{cX} 3)+\mathrm{h}(\mathrm{dX} 1+\mathrm{eX} 2+\mathrm{fX} 3)=(\mathrm{ga}+\mathrm{hd}) \mathrm{X} 1$ $+(g b+h e) X 2+(g c+h f) X 3$ where, $(g a+h d),(g b+h e)$ and (gc+hf) are the new coefficients of the re-encoded packet. The decoding operation is performed at the node V4 by collecting the coded packets. The coded packets are decoded by forming a matrix from linear coefficients. The matrix is referred to as decoding matrix or transfer matrix [21].

\subsubsection{Random Linear Network Coding for Multicast}

In this section, multicast network is considered with multiple independent messages, RLNC process for multicast is depicted in the Figure 15. The basic idea of RLNC for multicast communication can be illustrated in the Figure $17[28,1]$.

Multicast network $\mathrm{G}=(\mathrm{V}, \mathrm{E})$ is depicted in the Figure 14 , where $V$ is the set of nodes and $E$ is the set of edges. The source node V1 sending packets to nodes V4 and V5 with the help of forwarding nodes V2 and V3, the source node V1 observing three source packets X1, X2 and X3 also called native packets and randomly chosen coefficients $\alpha, \beta$, and $\gamma$ from finite field for encoding, there are three paths from V1 to V4 and another three paths from V1 to V5. Forwarding nodes V2 and V3 performs the re-encoding operation on the two received coded packets with the random coefficients, the coding vector of the new re-encoded packet can be given as input to the node V4 and V5. Decoding operation is performed at the node V4 and V5 by collecting the coded packets. These packets form linear equations and can be solved by forming a matrix which is referred as decoding matrix or transfer matrix. RLNC operations for multicasting is illustrated through the following equations, the information through the edges i.e e1, e2, e3 can be calculated by Equation (1),

$$
Y(e)=\sum_{l=1}^{\mu(v)} \alpha_{l, e} X(v, l)+\sum_{e^{\prime}: h e a d\left(e^{\prime}\right)=\operatorname{tail}(e)} \beta_{e^{\prime}, Y} Y\left(e^{\prime}\right)
$$

where $\mathrm{Y}(\mathrm{e})$ is coded packets on the outgoing edges from node $V_{1}$, which are linear combination of the sources $X(v, 1), X(v, 2), X(v, 3)$. The information at the destination nodes can be calculated by equation (2), which is received from forwarding nodes,

$$
Z(v, j)=\sum_{e^{\prime}: \operatorname{head}\left(e^{\prime}\right)=v} \varepsilon_{e^{\prime}, j} Y\left(e^{\prime}\right)
$$

where $\mathrm{Z}(\mathrm{v}, \mathrm{j})$ is re-encoded packet at destination nodes, which are received from forwarding nodes. Linear combinations of coded packets y (e1), y (e2), y (e3) on the edges e1, e2, and e3 can be expressed as,

$Y\left(e_{1}\right)=\alpha_{1, e_{1}} X(v, 1)+\alpha_{2, e_{1}} X(v, 2)+\alpha_{3, e_{1}} X(v, 3)$
$Y\left(e_{2}\right)=\alpha_{1, e_{2}} X(v, 1)+\alpha_{2, e_{2}} X(v, 2)+\alpha_{3, e_{2}} X(v, 3)$
$Y\left(e_{3}\right)=\alpha_{1, e_{3}} X(v, 1)+\alpha_{2, e_{3}} X(v, 2)+\alpha_{3, e_{3}} X(v, 3)$

Figure 14

RLNC in single source multicasting

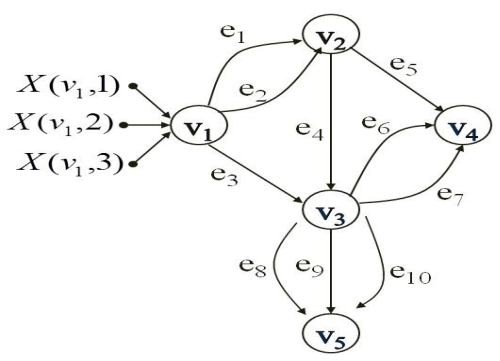

Source input: $X(v, l)=\left\{x_{0}(v, l), x_{1}(v, l), \ldots\right\}$

Information along the edges: $Y(e)=\left\{y_{0}(e), y_{1}(e), \ldots\right\}$

Sink output: $Z(v, l)=\left\{z_{0}(v, l), z_{1}(v, l), \ldots\right\}$

Where, $0,1,2 \ldots$ is time index 
Let $\alpha$ denote the $3 \times 3$ matrix. The above equations (3-5) can be written as matrix form as

$$
\left[\begin{array}{l}
Y\left(e_{1}\right) \\
Y\left(e_{2}\right) \\
Y\left(e_{3}\right)
\end{array}\right]=\alpha\left[\begin{array}{l}
x(v, 1) \\
x(v, 2) \\
x(v, 3)
\end{array}\right],
$$

where $\alpha=\left[\begin{array}{ccc}\alpha_{1, e_{1}} & \alpha_{2, e_{1}} & \alpha_{3, e_{1}} \\ \alpha_{1, e_{2}} & \alpha_{2, e_{2}} & \alpha_{3, e_{2}} \\ \alpha_{1, e_{3}} & \alpha_{2, e_{3}} & \alpha_{3, e_{3}}\end{array}\right]$

Linear combinations of re-encoded packets $\mathrm{y}\left(\mathrm{e}_{4}\right)$, $\mathrm{y}\left(\mathrm{e}_{5}\right), \mathrm{y}\left(\mathrm{e}_{6}\right), \mathrm{y}\left(\mathrm{e}_{7}\right)$ on the edges $\mathrm{e}_{4}, \mathrm{e}_{5}, \mathrm{e}_{6}$ and $\mathrm{e}_{7}$ can be expressed as,

$Y\left(e_{4}\right)=\beta_{e_{1}, e_{4}} Y\left(e_{1}\right)+\beta_{e_{2}, e_{4}} Y\left(e_{2}\right)$
$Y\left(e_{5}\right)=\beta_{e_{1}, e_{5}} Y\left(e_{1}\right)+\beta_{e_{2}, e_{5}} Y\left(e_{2}\right)$
$Y\left(e_{6}\right)=\beta_{e_{3}, e_{6}} Y\left(e_{3}\right)+\beta_{e_{4}, e_{6}} Y\left(e_{4}\right)$
$Y\left(e_{7}\right)=\beta_{e_{3}, e_{7}} Y\left(e_{3}\right)+\beta_{e_{4}, e_{7}} Y\left(e_{4}\right)$

Let $\beta$ denote the $3 \times 3$ matrix. The above equations (6-9) can be written as matrix form as

$$
\left[\begin{array}{l}
Y\left(e_{5}\right) \\
Y\left(e_{6}\right) \\
Y\left(e_{7}\right)
\end{array}\right]=\beta^{\prime} \cdot \alpha\left[\begin{array}{l}
x(v, 1) \\
x(v, 2) \\
x(v, 3)
\end{array}\right],
$$

where $\beta$ and $\alpha$ are given by

$$
\begin{aligned}
\beta & =\left[\begin{array}{ccc}
\beta_{e_{1}, e_{5}} & \beta_{e_{1}, e_{4}} \beta_{e_{4}, e_{6}} & \beta_{e_{1}, e_{4}} \beta_{e_{4}, e_{7}} \\
\beta_{e_{2}, e_{5}} & \beta_{e_{2}, e_{4}} \beta_{e_{4}, e_{6}} & \beta_{e_{2}, e_{4}} \beta_{e_{4}, e_{7}} \\
0 & \beta_{e_{3}, e_{6}} & \beta_{e_{3}, e_{7}}
\end{array}\right] \text { and } \\
\alpha & =\left[\begin{array}{lll}
\alpha_{1, e_{1}} & \alpha_{2, e_{1}} & \alpha_{3, e_{1}} \\
\alpha_{1, e_{2}} & \alpha_{2, e_{2}} & \alpha_{3, e_{2}} \\
\alpha_{1, e_{3}} & \alpha_{2, e_{3}} & \alpha_{3, e_{3}}
\end{array}\right]
\end{aligned}
$$

Destination node $\mathrm{V}_{4}$ recover the original packets from the received re-encoded packets $\left[\mathrm{y}\left(\mathrm{e}_{5}\right), \mathrm{y}\left(\mathrm{e}_{6}\right), \mathrm{y}\left(\mathrm{e}_{7}\right)\right]^{\mathrm{T}}$ and obtain,

$Z\left(v_{4}, 1\right)=\varepsilon_{e_{5}, 1} Y\left(e_{5}\right)+\varepsilon_{e_{6}, 1} Y\left(e_{6}\right)+\varepsilon_{e_{7}, 1} Y\left(e_{7}\right)$
$Z\left(v_{4}, 2\right)=\varepsilon_{e_{5}, 2} Y\left(e_{5}\right)+\varepsilon_{e_{6}, 2} Y\left(e_{6}\right)+\varepsilon_{e_{7}, 2} Y\left(e_{7}\right)$
$Z\left(v_{4}, 3\right)=\varepsilon_{e_{5}, 3} Y\left(e_{5}\right)+\varepsilon_{e_{6}, 3} Y\left(e_{6}\right)+\varepsilon_{e_{7}, 3} Y\left(e_{7}\right)$

For the destination node $\mathrm{V}_{5}$, Re-encoded data on edges $\mathrm{e}_{8}, \mathrm{e}_{9}$ and $\mathrm{e}_{10}$ denoted by $\mathrm{Y}\left(\mathrm{e}_{8}\right), \mathrm{Y}\left(\mathrm{e}_{9}\right)$ and $\mathrm{Y}\left(\mathrm{e}_{10}\right)$. They are linear combinations of $\mathrm{Y}\left(\mathrm{e}_{3}\right)$ and $\mathrm{Y}\left(\mathrm{e}_{4}\right)$ and can be expressed as,

$$
\left[\begin{array}{l}
Z\left(e_{8}\right) \\
Z\left(e_{9}\right) \\
Z\left(e_{10}\right)
\end{array}\right]=\left[\begin{array}{cc}
\gamma e_{3} e_{8} & \gamma e_{4} e_{8} \\
\gamma e_{3} e_{9} & \gamma e_{4} e_{9} \\
\gamma e_{3} e_{10} & \gamma e_{4} e_{10}
\end{array}\right]\left[\begin{array}{l}
Y\left(e_{3}\right) \\
Y\left(e_{4}\right)
\end{array}\right]
$$

By applying equation (6) in above expression and obtain the following, because, both coded packets $\mathrm{y}\left(\mathrm{e}_{1}\right)$ and $\mathrm{y}\left(\mathrm{e}_{2}\right)$ pass through edge $\mathrm{e}_{4}$, therefore, disjoint paths are not enough for $V_{5}$ from $V_{1}$.

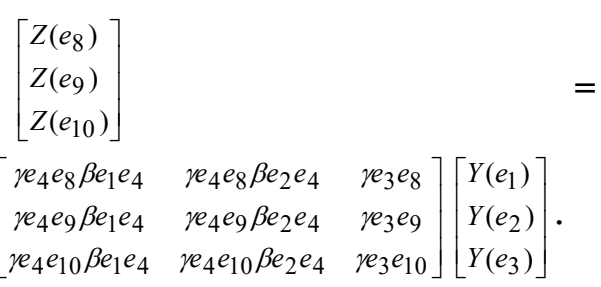

Further, $\left[\mathrm{Y}\left(\mathrm{e}_{1}\right) \mathrm{Y}\left(\mathrm{e}_{2}\right) \mathrm{Y}\left(\mathrm{e}_{3}\right)\right]^{\mathrm{T}}$ can be represented in terms of $[\mathrm{X}(\mathrm{v}, 1) \mathrm{X}(\mathrm{v}, 2) \mathrm{X}(\mathrm{v}, 3)]^{\mathrm{T}}$ and denote the above matrix by $\kappa$, which share the common coefficients of $\mathrm{y}\left(\mathrm{e}_{1}\right)$ and $\mathrm{y}\left(\mathrm{e}_{2}\right)$. Then,

$\left[\begin{array}{l}Z\left(e_{8}\right) \\ Z\left(e_{9}\right) \\ Z\left(e_{10}\right)\end{array}\right]=\kappa \cdot \alpha \cdot\left[\begin{array}{l}x(v, 1) \\ x(v, 2) \\ x(v, 3)\end{array}\right]$.

Destination node $V_{5}$ recover the original packets from the received re-encoded packets $\left[y\left(e_{8}\right), y\left(e_{9}\right), y\left(e_{10}\right)\right]^{T}$ and obtain,

$$
Z\left(v_{5}, 1\right)=\gamma_{e_{8}, 1} Y\left(e_{8}\right)+\gamma_{e_{9}, 1} Y\left(e_{9}\right)+\gamma_{e_{10}, 1} Y\left(e_{10}\right)
$$

$Z\left(v_{5}, 2\right)=\gamma_{e_{8}, 2} Y\left(e_{8}\right)+\gamma_{e_{9}, 2} Y\left(e_{9}\right)+\gamma_{e_{10}, 2} Y\left(e_{10}\right)$

$Z\left(v_{5}, 3\right)=\gamma_{e_{8}, 3} Y\left(e_{8}\right)+\gamma_{e_{9}, 3} Y\left(e_{9}\right)+\gamma_{e_{10}, 3} Y\left(e_{10}\right)$

Now, the original multicast data at destination node $\mathrm{V}_{4}$ is decoded from equations (10) - (12), similarly, destination node V5 is decoded from equations (13)(15), by solving the relation between $\bar{x}$ and $\bar{z}$,

$$
\bar{z}=\bar{x} \cdot M
$$

where $\bar{x}$ and $\bar{z}$ are input and ouput process vectors respectively, $\mathrm{M}$ is the transfer matrix, which is obtained from solving the following matrix, 
Figure 15

Flowchart for RLNC for multicast

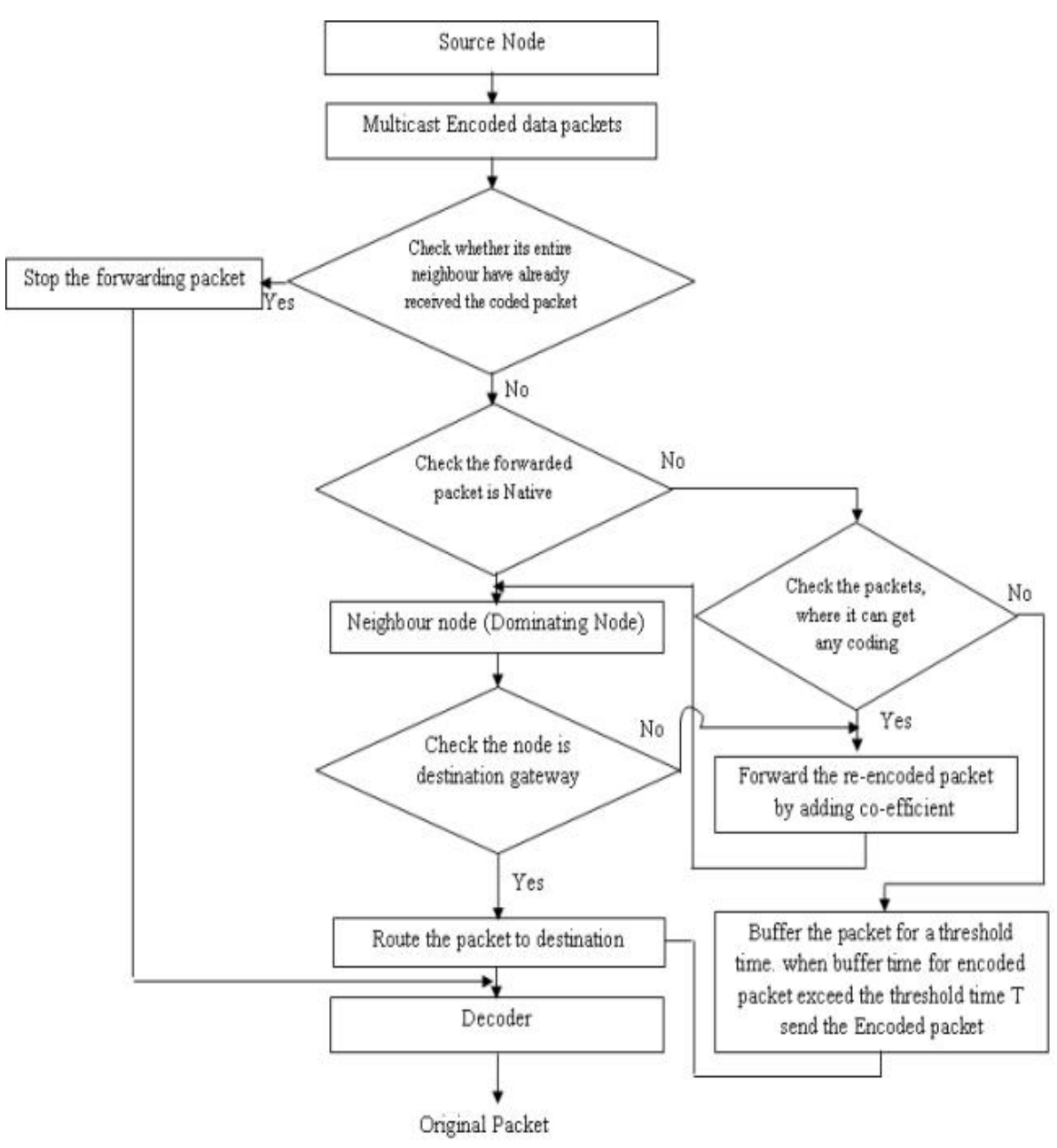

$$
M=A \cdot\left[\begin{array}{ccc}
\beta_{e_{1}, e_{5}} & \beta_{e_{1}, e_{4}} \beta_{e_{4}, e_{6}} & \beta_{e_{1}, e_{4}} \beta_{e_{4}, e_{7}} \\
\beta_{e_{2}, e_{5}} & \beta_{e_{2}, e_{4}} \beta_{e_{4}, e_{6}} & \beta_{e_{2}, e_{4}} \beta_{e_{4}, e_{7}} \\
0 & \beta_{e_{3}, e_{6}} & \beta_{e_{3}, e_{7}}
\end{array}\right] \cdot B
$$

where,

$A=\left[\begin{array}{ccc}\alpha_{1, e_{1}} & \alpha_{1, e_{2}} & \alpha_{1, e_{3}} \\ \alpha_{2, e_{1}} & \alpha_{2, e_{2}} & \alpha_{2, e_{3}} \\ \alpha_{3, e_{1}} & \alpha_{3, e_{2}} & \alpha_{3, e_{3}}\end{array}\right] \quad B=\left[\begin{array}{ccc}\varepsilon_{e_{5}, 1} & \varepsilon_{e_{5}, 2} & \varepsilon_{e_{5}, 3} \\ \varepsilon_{e_{6}, 1} & \varepsilon_{e_{6}, 2} & \varepsilon_{e_{6}, 3} \\ \varepsilon_{e_{7}, 1} & \varepsilon_{e_{7}, 2} & \varepsilon_{e_{7}, 3}\end{array}\right]$
The square matrices $\beta^{\prime} . \alpha$ and $\kappa . \alpha$ are invertible and unicoding is possible. Each destination node wants to decode the vector data $\bar{z}$. This implies that de$\mathrm{t}(\beta . \alpha) \neq 0$ and $\operatorname{det}(\kappa . \alpha) \neq 0 \operatorname{det}\left(\mathrm{M}_{\mathrm{i}}\right) \neq 0 \quad \forall_{\mathrm{i}}$, therefore the product of determinant is non-zero. Determinant is non-zero means that it has some data for the particular destination node.

\subsubsection{Operations of Proposed Protocol RLNMCDS-ODMRP}

Implementation of the proposed protocol has two phases as shown in Figures 19 and 20. First, source 
node discovers route and constructs MCDS using convex hull, Second, source node transmitting data by applying RLNC through constructed MCDS in ODM$\mathrm{RP}$ to its receivers.

The first phase is shown in Figure 16, where source node broadcasting join request packet JOIN_REQUEST as route construction process, source node check whether it is a source gateway host, if so, which act as a new source and check this node belong to network or adjacent to source node, if yes, mark the selected node as dominating node, otherwise select the another routing path to find dominating node as above steps, then the dominating node collect neighbor information of its neighbor node and member list. Based on the neighborhood information and member list, the dominating node rebroadcast the JOIN_REQUEST packet to its neighbor, then the neighbor node checks the above condition to find dominating node, finally check whether the selected node is destination gateway, if so, it construct and forward join reply packet JOIN_REPLY to source node through dominating nodes. In the second phase as shown in Figure 17, Source node multicast encoded data packets to its receivers through the dominating node (forwarding node) of MCDS, based on the neighborhood information it checks whether it's entire neighbor already have received coded packet, if yes, it simply stops the forwarding packet and checks whether it is a native packet. If yes, coded packets are sends directly to its neighboring dominating node. If not, dominating node forward the re-encoded packet by adding co-efficient to its adjacent dominating node and the re-encoded packets are buffered for a threshold time $\mathrm{T}$ before transmit.

\section{Figure 16}

Route Discovery and Route reply through MCDS

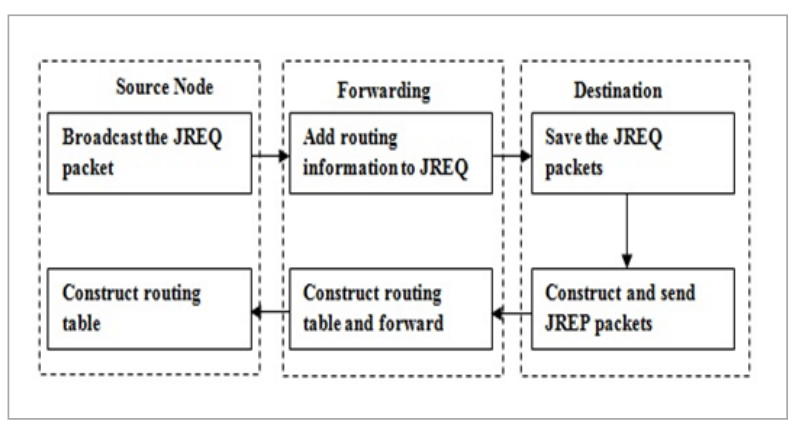

Figure 17

Multicast Data Transmission by applying RLNC

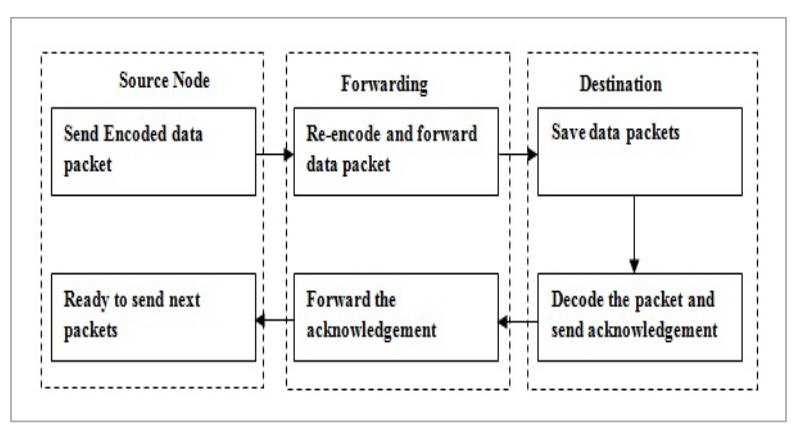

When buffer time for the encoded packet exceeds the threshold time $\mathrm{T}$ send the encoded packet to its destination node. If the receiving node is destination node, which save and decodes original multicast data. Then acknowledgement is send to the source node through connected dominating nodes. Otherwise, forward the re-encoded packet to its neighbor node until the re-encoded packet reached to its destination node, now the source node is ready to multicast next packet to its receivers.

\section{Simulation Environment and Performance Evaluation}

\subsection{Random Linear Network Coding}

Nodes were positioned evenly at indiscriminate places over an area of $500 \mathrm{~m} \times 500 \mathrm{~m}$ in the simulation $\mathrm{ex}$ periment. The multicast traffic is Constant Bit Rate (CBR) having 250 bytes data packet. The simulation set-ups are generated using the set dest network simulator-2 (NS-2) with simulation time of 200 seconds. Mobility model employs a random waypoint in a rectangular field. At this time, 1-to-many multicast approach was considered, i.e., Sender is sticked to be one, then receiver may change in span of 9 and 99 . The least and greatest speed range between 0 and $20 \mathrm{~m} / \mathrm{s}$, correspondingly in pause time interval is 1 simulation seconds, that relates to steady motion as well as transmission rate is $128 \mathrm{Kbps}$, transmission range is $50 \mathrm{~m}$ for all nodes. The simulation parameters are summarized in Table 1. 
Table 1

Simulation Parameters

\begin{tabular}{|c|c|c|}
\hline S.No & Parameters & Particulars \\
\hline 1. & Routing protocol & ODMRP \\
\hline 2. & Simulator & NS-2 \\
\hline 3. & No.of nodes & 100 \\
\hline 4. & Simulation time & 200 secs \\
\hline 5. & Simulation area & $\begin{array}{l}500 \mathrm{~m} \times 500 \mathrm{~m} \text { to } \\
1200 \mathrm{~m} \times 1200 \mathrm{~m}\end{array}$ \\
\hline 6. & Node movement & Random way point \\
\hline 7. & Sender \& Receiver & $\begin{array}{l}\text { Sender-1 } \\
\text { Recevier-09-99 }\end{array}$ \\
\hline 8. & Pause time & $1 \mathrm{sec}$ \\
\hline 9. & Traffic & CBR \\
\hline 10. & CBR Packet size & 250 bytes \\
\hline 11. & Traffic Load & $5 \mathrm{pkts} / \mathrm{sec}$ \\
\hline 12. & Arrival Rate & 10 kbps-100 kbps \\
\hline 13. & Routing Metric & $\begin{array}{l}\text { Success Probability } \\
\text { Product (SPP) }\end{array}$ \\
\hline 14. & Mobility speed & $0,5,10,15,20 \mathrm{~m} / \mathrm{s}$ \\
\hline 15. & Transmission rate & $128 \mathrm{Kbps}$ \\
\hline 16. & Transmission range & $50 \mathrm{~m}$ \\
\hline 17. & Topology & Multi-hop \\
\hline 18. & Methods & MCDS and RLNC \\
\hline
\end{tabular}

\subsection{Performance Metrics}

\subsubsection{Reliability}

The ability of successful end-to-end data delivery ratio is called as reliability $[25,5]$.

$\operatorname{Reliability}\left(\mathrm{r}_{0}, \mathrm{r}_{1} \ldots . \mathrm{r}_{\mathrm{h}-1}, \mathrm{r}_{\mathrm{h}}\right)=\exp \left(-\sum_{\mathrm{i}=1}^{\mathrm{h}} \frac{\mathrm{d}_{\mathrm{ri}-1 \mathrm{ri}}^{\mathrm{k}}}{\mathrm{snr}_{\mathrm{ri}-1 \mathrm{ri}}}\right)$,

where $r_{0}, r_{1} \ldots r_{h-1}, r_{h}$ are route between source and destination nodes; $d_{r i-1 r i}^{k}$ is the distance between the neighboring nodes; $s n r_{r i-1 r i}$ is the signal-to-noise power for the transmitted signal

\subsubsection{Experimental Results and Analysis}

Experimental results and analysis of the proposed approach (RLNMCDS-ODMRP) for the performance metric of reliability are elaborated in this section. RLNMCDS-ODMRP is simulated and its performance is analyzed by the following four scenarios: i) By varying terrain size, ii) By varying arrival rate, iii) By varying number of nodes and iv) By varying mobility speed. The following graphs show that performance comparison between proposed protocol RLNMCDS-ODMRP, ODMRP with RLNC, ODMRP with MCDS and normal ODMRP.

\section{Scenario-I - By varying terrain size}

In the scenario-I, the performance of proposed protocol is measured for the reliability considered in this paper by varying the terrain size from $500 \mathrm{~m} \times 500 \mathrm{~m}$ to $1200 \mathrm{~m} \times 1200 \mathrm{~m}$ for the fixed 20 nodes, which are uniformly deployed in the network coverage area at minimum speed of $0 \mathrm{~m} / \mathrm{s}$, selected routing metric is $\mathrm{SPP}$, arrival rate is set to $10 \mathrm{kbps}$ and traffic load is fixed as 5pkts/sec and equally distributed among all senders. Transmission range of a sensor node is fixed at 50m. It is shown in Figure 18.

After node deployment, maintenance of node is a difficult process. For this reason, all nodes must adapt their behaviors to the environmental changes. Sensor nodes are having the static feature and it knows the coordinates of their location. The location co-ordinates for the sensors are allocated at the beginning itself. In this method nodes are deployed in Unit Disk (UD) model.

\section{Scenario-II - By varying arrival rate}

In the scenario-II, the performance of proposed protocol is measured for the reliability considered in this work by varying the multicast messages arrival rate from $10 \mathrm{kbps}$ to $100 \mathrm{kbps}$ for a static speed of $0 \mathrm{~m} / \mathrm{s}$ and for the fixed 20 nodes network coverage area, selected routing metric is SPP, terrain size is fixed as $500 \mathrm{~m} \times 500 \mathrm{~m}$, traffic load is fixed as 5 pkts/sec and equally distributed among all senders.

Message arrival rate is expressed as average number of multicast messages arrived during unit of time, which affects protocol performance. A sender generated multicast traffic based on a deterministic model of one-active arrival pattern, multicasting nodes are started their multicasting at $0.5 \mathrm{~s}$ after joining the group and continued for a period of 10 s for a given arrival rate.

Figure 19 shows the reliability vs message arrival rate (kbps). First, the proposed protocol RLN- 
MCDS-ODMRP delivers high reliability for low arrival rate, which states that the proposed protocol is able to recover lost messages for low arrival rate. RLNC-ODMRP gives slightly higher reliability than MCDS-ODMRP under low arrival rate. Second, reliability starts decreasing significantly when arrival rate or network congestion is increased after a threshold for other protocols, because which consumes significantly larger bandwidth.

\section{Scenario-III - By varying number of nodes}

In the third scenario, the performance of proposed protocol is measured for the reliability considered in this work by increasing number of nodes from 09 to 99 nodes for a static speed of $0 \mathrm{~m} / \mathrm{s}$ in network coverage area and selected routing metric is SPP. Arrival rate is set to $10 \mathrm{kbps}$, terrain size is fixed as $500 \mathrm{~m}$ $\times 500 \mathrm{~m}$, traffic load is fixed as $5 \mathrm{pkts} / \mathrm{sec}$ and equally distributed among all senders.

Reliability should be high for better performance of the network. Reliable protocol consumes less bandwidth by reducing the retransmission and acknowledgement of participating nodes in a network. As described in the Figure 20, efficient techniques can be used to improve the reliability. To achieve certain reliability, limited number of transmission, neighborhood nodes estimation and channel quality link between source and destination node is important.

In the single source multicasting, collisions between multicast packets are very rare under low load therefore, reliability increases for all protocol. From Figure 21, it is observed that, when number of nodes are getting increased, the proposed protocol RLNMCDS-ODMRP provides good reliability when compare to other multicast protocols because, it reduces packet error rate in end-to-end in dynamic environment. ODMRP with RLNC offers average reliability because of communication overhead. As nodes are strongly connected in ODMRP with MCDS, the reliability is improved than Normal_ODMRP.

\section{Scenario-IV - By varying speed of the nodes}

In the Scenario-IV, the performance of proposed protocol is measured for the reliability considered in this work. It is computed by enlarging the speed of the nodes from 0 to $20 \mathrm{~m} / \mathrm{s}$ for the static 20 nodes in network coverage area and selected routing metric is SPP. Arrival rate is set to $10 \mathrm{kbps}$, terrain size is fixed as $500 \mathrm{~m} \times 500 \mathrm{~m}$, traffic load is fixed as $5 \mathrm{pkts} / \mathrm{sec}$ and equally distributed among all senders.
Figure 18

Terrain size (m) vs Reliability

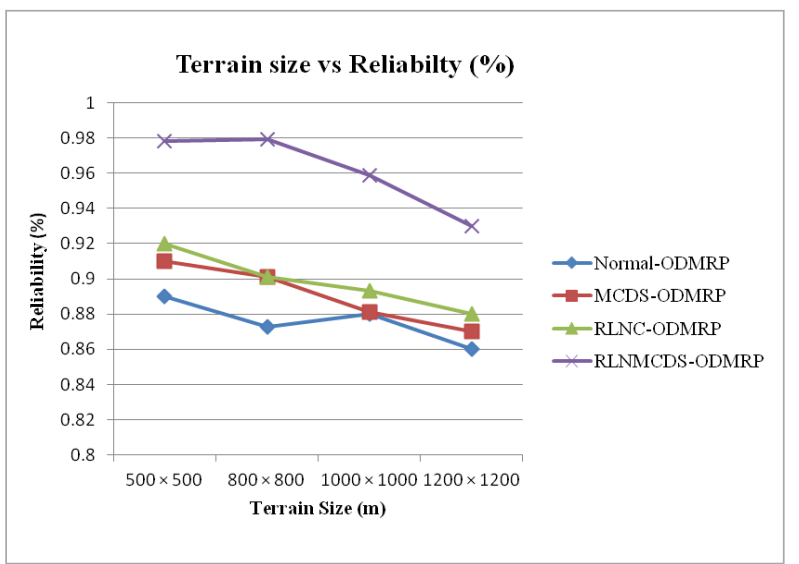

Figure 19

Arrival rate (kbps) vs Reliability

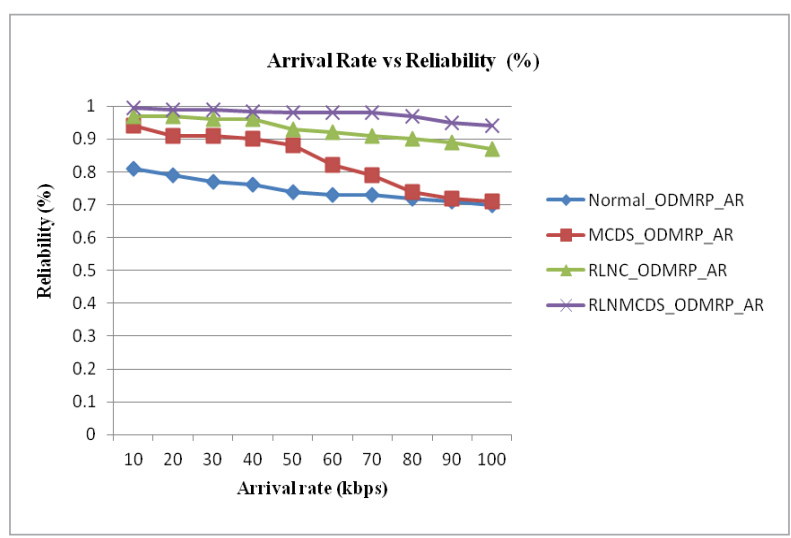

Figure 20

Mobility speed (m/s) vs Reliability

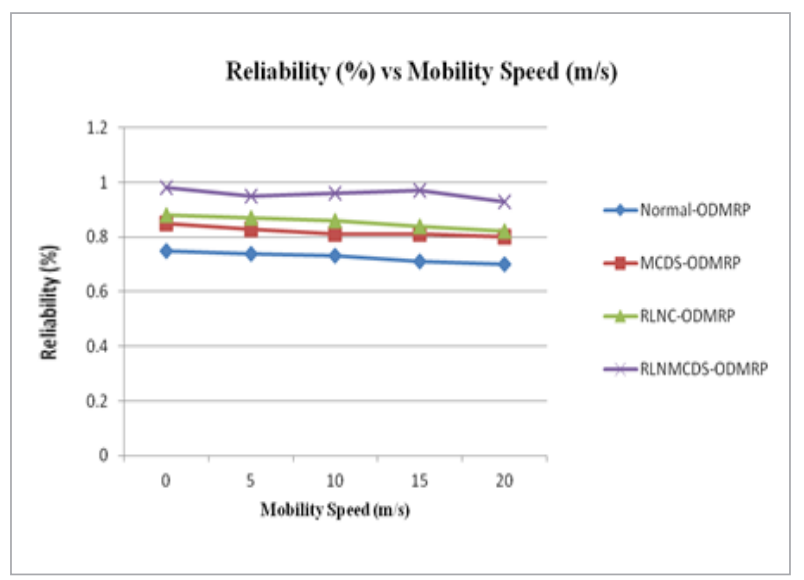




\section{Figure 21}

Number of nodes vs Reliability

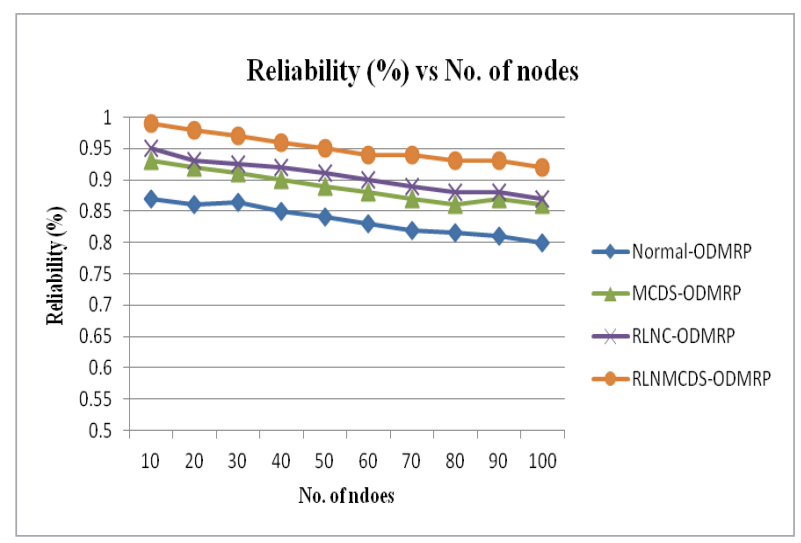

When nodes are moving in a coverage area at different speed, it constructs multiple independent paths from source to destinations dynamically. This ensures that reliability of a network. Non-acknowledged virtual backbone multicasting is improving the reliability of network. Figure 21 shows that the reliability versus mobility speed $(\mathrm{m} / \mathrm{s})$.

\section{References}

1. Aditya, R., Shi, J., Wesel, R. D. On the Capacity of Network Coding for Random Networks. IEEE Transactions on Information Theory, 2005, 51, 2878-2885. https://doi.org/10.1109/TIT.2005.851725

2. Ahlswede, R., Cai, N., Li, S.-Y. R., Yeung, R. W. Network Information Flow. IEEE Transactions on Information Theory, 2000, 46, 1204-1216, https://doi. org/10.1109/18.850663

3. Akyildiz, I.F., Su, W., Sankarasubramaniam, Y., Cayirci, E. Survey on Sensor Networks. IEEE Comm. Magazine, 2002, 40(8), 102-114. https://doi.org/10.1109/ MCOM.2002.1024422

4. Al-Nabhan, N., Zhang, B., Al-Rodhaan, M., Al-Dhelaan, A. Two Connected Dominating Set Algorithms for Wireless Sensor Networks. Wireless Algorithms, Systems, and Applications, Lecture Notes in Computer Science, 2012, 74(5), 705-713. https://doi.org/10.1007/978-3642-31869-6_62

5. Anisi, M. H., Abdullah, A. H., Razak, S. A. Energy-efficient and Reliable Data Delivery in Wireless Sensor
It is observed that, on improving the speed of nodes, the proposed protocol RLNCDS-ODMRP provides good reliability since the transmission delay is very low. On the other hand, ODMRP with RLNC, ODMRP with MCDS and normal ODMRP has less reliability since the delay is high.

\section{Conclusion}

In this paper, Random Linear Network Coding over Minimum Connected Dominating Set in ODMRP (RLNMCDS-ODMRP) is proposed to improve the reliability in multicast routing protocol for WSN. Based on the experiments, it is concluded that the proposed protocol RLNMCDS-ODMRP has following advantages, (1) consumes less time to construct multicast topology than normal ODMRP, (2) reliability is enhanced 12 times of its conventional ODMRP, (3) achieves $95 \%$ of the theoretical maximum multicast capacity which is several times of ODMRP's, meanwhile only with about $15 \%$ extra bandwidth consumption compared with ODMRP.

Networks. Wireless Networks, 2013, 19(4), 495-505. https://doi.org/10.1007/s11276-012-0480-x

6. Bai, X., Zhao, D., Bai, S., Wang, Q., Li, W., Mu, D. Minimum connected Dominating Sets in Heterogeneous 3D Wireless Ad Hoc Networks. Ad Hoc Networks, 97, 102023. ISSN: 1570-8705. https://doi.org/10.1016/j. adhoc.2019.102023

7. Blum, J., Ding, M., Thaeler, A., Cheng, X. Connected Dominating Set in Sensor Networks and MANETs. Handbook of Combinatorial Optimization, 2005, 5, 329-369. https://doi.org/10.1007/0-387-23830-1_8

8. Dong, J., Curtmola, R., Nita-Rotaru. C. Secure High-Throughput Multicast Routing in Wireless Mesh Networks. IEEE Transactions on Mobile Computing, 2011, 10(5). https://doi.org/10.1109/TMC.2010.194

9. Du, H., Wu, W., Shan, S., Kim, D., Lee, W. Constructing Weakly Connected Dominating Set for Secure Clustering in Distributed Sensor Network. Journal of Combinatorial Optimization, 2012, 23(2), 301-30\%. https://doi. org/10.1007/s10878-010-9358-y 
10. Dumitrescu, S., Shao, M., Wu, X. Layered Multicast with Interlayer Network Coding. The 28th IEEE Conference on Computer Communications (INFOCOM'09), 2009. https://doi.org/10.1109/TMM.2010.2095833

11. El Gamal, A., Kim, Y.-H. Network Information Theory. Cambridge University Press, New York, USA, 2012; https://doi.org/10.1017/CBO9781139030687

12. Ghafouri, A., Ghasemi, A. A Power-based Method for Improving the ODMRP Protocol Performance in Mobile Ad-hoc Networks. International Journal of Wireless and Microwave Technologies, 2018, 8(2), 27-36. https:// doi.org/10.5815/ijwmt.2018.02.03

13. Guha, S., Khuller, S. Approximation Algorithms for Connected Dominating Sets. Algorithmica, 1998, 20(4), 374-387. https://doi.org/10.1007/PL00009201

14. Han, Z.-J., Wang, r.-C., Xiao, F. A Multicast Algorithm for Wireless Sensor Networks Based on Network Coding. International Journal of Distributed Sensor Networks, 2014. https://doi.org/10.1109/AIMSEC.2011.6010849

15. Haynes, T. W., Hedetniemi, S., Slater, P. Fundamentals of Domination in Graphs. Marcel Dekker, Inc., A Series of Monographs and Textbooks, 1998.

16. Heide, J., Shi, S., Fouli, K., Cook, V. Random Linear Network Coding (RLNC): Background and Practical Considerations. Feb 2019. https://www.ietf.org/archive/id/ draft-heide-nwcrg-rlnc-background-00.txt.

17. Ho, T., Médard, M., Koetter, R., Karger, D. R., Effros, M., Shi, J., Leong, B. A Random Linear Network Coding Approach to Multicast. IEEE Transactions on Information Theory, 2006, 52(10). https://doi.org/10.1109/ TIT.2006.881746

18. Hongwei Du; Qiang Ye; Jioafei Zhong; Yuexuan Wang; Wonjun Lee; Haesun Park. PTAS for Minimum Connected Dominating Set with Routing Cost Constraint in Wireless Sensor Networks. International Journal of Distributed Sensor Networks, 2013. https://doi.org/10.1016/j.tcs.2004.08.013

19. Jaggi, S., Sanders, P., Chou, P. A., Effros, M., Egner, S., Jain, K., Tolhuizen, L. M. G. M. Polynomial Time Algorithms for Multicast Network Code Construction. IEEE Transactions on Information Theory, 2005, 51(6), 19731982. https://doi.org/10.1109/TIT.2005.847712

20. Jennings, M. V. The Application of Network Coding to Multicast Routing in Wireless Networks Diss. Massachusetts Institute of Technology, 2007. http://hdl.handle.net/1721.1/40532

21. Kashif, M., Kunz, T., Matrawy, A. Adaptive Random Linear Network Coding with Controlled Forwarding for
Wireless Broadcast. Wireless Days, 2010. https://doi. org/10.1109/WD.2010.56577753

22. Katti, S., Rahul, H., Hu, W., Katabi, D., Médard, M., Crowcroft, J. XORs in the Air: Practical Wireless Network Coding. IEEE/ACM Transactions on Networking, 2008, 497-510. https://doi.org/10.1109/TNET.2008.923722

23. Keller, L., Atsan, E., Argyraki, K., Fragouli, C. SenseCode: Network Coding for Reliable Sensor Networks. ACM Transactions on Sensor Networks (TOSN), 2013, 9(2). https://doi.org/10.1145/2422966.2422982

24. Keshavarz-Haddad, A., Riedi, R. H. Bounds on the Benefit of Network Coding for Wireless Multicast and Unicast. IEEE Transactions on Mobile Computing, 2014, 13(1), 102-115. https://doi.org/10.1109/TMC.2012.234

25. Khandani, A., Modiano, E., Abounadi, J., Zheng, L. Reliability and Route Diversity in Wireless Networks. Proceedings of the Conference on Information Science and System, 2005. https://doi.org/10.1109/T-WC.2008.070770

26. Kui, X., Sheng, Y., Du, H., Liang, J. Constructing a CDS-based Network Backbone for Data Collection in Wireless Sensor Networks. International Journal of Distributed Sensor Networks, 2013. https://doi. org/10.1155/2013/258081

27. Lee, S. J., Su, W., Gerla, M. On-demand Multicast Routing Protocol in Multihop Wireless Mobile Networks. ACM/Kluwer Mobile Networks and Applications, 2002, 7(6), 441-452. doi.org:10.1023/A:1020756600187

28. Li, S. R., Yeung, R. W., Cai, N. Linear Network Coding. IEEE Transactions on Information Theory, 2003, 371381. https://doi.org/10.1109/TIT.2002.807285

29. Li, W., Lu, K., Wang, J., Huang, L., Wu, D. O. On the Throughput Capacity of Wireless Sensor Networks with Mobile Relays. IEEE Transactions on Vehicular Technology, 2012, 61(4), 1801-1809. https://doi.org/10.1109/ TVT.2012.2188145

30. Liang, O. Multipoint Relay and Connected Dominating Set Based Broadcast Algorithms for Wireless Ad Hoc Networks. Monash University, Melbourne, $200 \%$.

31. Lien, C.-M., Chang, C.-S., Lee, D.-S. A Universal Stabilization Algorithm for Multicast Flows with $\mathrm{Ne}$ twork Coding. IEEE Transactions on Communications, 2013, 61(2), 712-721. https://doi.org/10.1109/ TCOMM.2012.102512.120046

32. Lin, K. C.-J., Yang, D.-N. Multicast with Intraflow Network Coding in Multirate Multichannel Wireless Mesh Networks. IEEE Transactions on Vehicular Technology, 2013, 62(8), 3913-3927. https://doi.org/10.1109/ TVT.2013.2260350 
33. Lisiecki, D., Zhang, P., Theel, O. CONE: A Connected Dominating Set-Based Flooding Protocol for Wireless Sensor Networks. Sensors 2019, 19, 2378. https://doi. org/10.3390/s19102378

34. Mallapur, S. V., Patil, S. R., Agarkhed, J.V. Multi-constraints Reliable Multicast Routing Protocol for Group Communications in MANETs. International Journal of Information Technology, 2018, 10, 147. https://doi. org/10.1109/COMSNETS.2016.7439967

35. Ostovari, P., Wu, J., Khreishah, A. Network Coding Techniques for Wireless and Sensor Networks. In Ammari, H. M. (Ed.), The Art of Wireless Sensor Networks, 2013, Springer. https://doi.org/10.1007/978-3-642-40009-4_5

36. Purohit, G. N., Sharma, U. Constructing Minimum Connected Dominating Set: Algorithmic Approach. International Journal on Applications of Graph Theory in Wireless Ad Hoc Networks and Sensor Networks, 2010, 2. https://doi.org/10.5121/jgraphoc.2010.2305

37. Rahman, A., Anwar, F. A Simulation Based Performance Comparison of Routing Protocol on Mobile Ad-hoc Network (Proactive, Reactive and Hybrid). Proceedings of the IEEE ICCCE, 2010. https://doi.org/10.1109/ICCCE.2010.5556751

38. Rai, M., Verma, S., Tapaswi, H. A Power Aware Minimum Connected Dominating Set for Wireless Sensor Networks. Journal of Networks, 2009, 4(6), 511-519. https://doi.org/10.4304/jnw.4.6.511-519

39. Rout, R. R., Ghosh, S. K. Enhancement of Lifetime Using Duty Cycle and Network Coding in Wireless Sensor Networks. IEEE Transactions on Wireless Communications, 2013, 12(2), 656-667. https://doi.org/10.1109/ TWC.2012.111412.112124

40. Schumacher, A. Dominating Set Based Routing, Algorithms for Ad-hoc Networking. Seminar talk, 2003. https://doi.org/10.1002/0471224561

41. Sohel, S. R., Ferdous, C. N., Rahman, A., Nafis, A. J., Arabi, S., Rab, R. On Constructing Contention Aware Connected Dominating Sets (CDS) for Inter Connectivity Among Internet of Things (IoT) Devices. International Journal of Multimedia Intelligence and Security, 2019, 3(3). https://doi.org/10.1504/IJMIS.2019.104795

42. Sun, X., Yang, Y., Ma, M. Minimum Connected Dominating Set Algorithms for Ad Hoc Sensor Networks. Sensors 2019, 19, 1919. https://doi.org/10.3390/s19081919

43. Swapna, B. T., Eryilmaz, A., Shroff, N. B. Throughput-Delay Analysis of Random Linear Network Coding for Wireless Broadcasting. IEEE Transactions on Informati- on Theory, 2013, 59(10), 6328-6341. arXiv:1101.3724. https://doi.org/10.1109/TIT.2013.22r1895

44. Torkestani, J. A., Meybodi, M. R. Mobility-based Multicast Routing Algorithm for Wireless Mobile Ad-hoc Networks. A Learning Automata Approach Computer Communications, 2010, 33(6), 721-735. https://doi.org/10.1016/j.comcom.2009.11.019

45. Torkestani, J. A., Meybodi, M. R. Weighted Steiner Connected Dominating Set and Its Application to Multicast Routing in Wireless MANETs. Wireless Personal Communication, Springer, 2010. https://doi. org/10.1007/s1127\%-010-9936-4

46. Tran, T. T., Tang, X., Xie, B. Secure Wireless Multicast for Delay-Sensitive Data via Network Coding. IEEE Transactions on Wireless Communications, 2013, 12(7), 3372-3387. https://doi.org/10.1109/ TWC.2013.062413.121557

47. Tsimbalo, E., Sandell, M. Reliability of Relay Networks Under Random Linear Network Coding. IEEE Transactions on Communications, 2019, 67(8), 5230-5240. https://doi.org/10.1109/TCOMM.2019.2911578

48. Wang, S., Wang, C., Peng, K., Tan, G., Jiang, H., Dong, Y. Network Coding over Connected Dominating Set: Energy Minimal Broadcasting in Wireless Ad Hoc Networks. Wireless Networks, 2014, 20(5). https://doi. org/10.1007/s11276-013-0648-z

49. Wang, X. M., Wang, J. P., Xu, Y. L. Data Dissemination in Wireless Sensor Networks with Network Coding. EURASIP Journal on Wireless Communications and Networking, 2010, Article Id 465915. https://doi. org/10.1155/2010/465915

50. West, D. B. Introduction to Graph Theory, 2nd ed. Upper Saddle River, NJ: Prentice Hall, 2001.

51. Zeng, D., Guo, S., Xiang, Y., Jinl, H. On the Throughput of Two-Way Relay Networks Using Network Coding. IEEE Transactions on Parallel and Distributed Systems, 2014, 25(1), 191-199. https://doi.org/10.1109/ TPDS.2013.187

52. Zhang, Z., Gao, X., Wu, W., Du, D. Z. A PTAS for Minimum Connected Dominating Set in 3-Dimensional Wireless Sensor Networks. Journal of Global Optimization, 2009, 45(3), 451-458. https://doi.org/10.1007/ s10898-008-9384-9

53. Zhu, Y., Li, B., Guo, J. Multicast with Network Coding in Application Layer Overlay Networks. IEEE Journal on Selected Areas in Communications, 2004, 22(1), 107120. https://doi.org/10.1109/JSAC.2003.818801 